Instituto Internacional de Investigación y Desarrollo Tecnológico Educativo INDTEC, C.A.

DOI: https://doi.org/10.29394/Scientific.issn.2542-2987.2021.6.19.13.264-278

OAI-PMH: http://www.indteca.com/ojs/index.php/Revista Scientific/oai

Ensayo Original / Original Essay

\title{
Acercamiento a la realidad educativa en las escuelas del sector rural venezolano
}

Autora: Adriana Hernández Lobo Universidad Pedagógica Experimental Libertador, UPEL fatimavalentina 22@hotmail.com Mérida, Venezuela https://orcid.org/0000-0003-4974-3465

\section{Resumen}

El ensayo precisa algunos elementos presentes en la realidad educativa de las escuelas del sector rural venezolano: la constitución heterogénea de los grupos en el aula de clase, la descontextualización curricular, la fragmentación y desvinculación de los contenidos enseñados, el desempeño docente en la ruralidad y su formación. El objetivo del ensayo es colocar de manifiesto cual es la realidad del proceso de enseñanza y aprendizaje en el sector rural, y como debería de ser obedeciendo a las particularidades del contexto, para lograr la formación integral de los estudiantes y favorecer el arraigo y pertinencia socio- cultural de sus habitantes. En tal sentido, es necesario hablar de renovación pedagógica en las escuelas rurales, caracterizada por un proceso especial, cuyo interés esté centrado en las necesidades de los estudiantes; se trata de incorporar en el aula, en la escuela, la familia y la comunidad todos aquellos factores fundamentales para desarrollar un currículo enmarcado en una educación de calidad. Por tanto, la enseñanza en las escuelas del sector rural debe responder a un acto humanista integrando la realidad de cada estudiante con el propósito de la educación, que conduzca a la formación libre e independiente de cada ser.

Palabras clave: realidad educativa; escuelas rurales; descontextualización curricular; desempeño docente rural.

Cómo citar este ensayo:

Hernández, A. (2021). Acercamiento a la realidad educativa en las escuelas del sector rural venezolano. Revista Scientific, 6(19), 264-278, e-ISSN: 2542-2987. Recuperado de: https://doi.org/10.29394/Scientific.issn.2542-2987.2021.6.19.13.264-278

Fecha de Recepción: 11-09-2020
Fecha de Aceptación:

04-12-2020
Fecha de Publicación: 05-02-2021 


\title{
Approach to the educational reality in schools in the Venezuelan rural sector
}

\begin{abstract}
The essay specifies some elements present in the educational reality of the schools of the Venezuelan rural sector: the heterogeneous constitution of the groups in the classroom, the curricular decontextualization, the fragmentation and disconnection of the taught contents, the teaching performance in rural areas and their training. The objective of the essay is to show what is the reality of the teaching and learning process in the rural sector, and how it should be obeying the particularities of the context, to achieve the integral formation of students and favor the roots and social relevance - cultural of its inhabitants. In this sense, it is necessary to speak of pedagogical renewal in rural schools, characterized by a special process, whose interest is centered on the needs of the students; it is about incorporating in the classroom, school, family and community all those fundamental factors to develop a curriculum framed in a quality education. Therefore, teaching in schools in the rural sector must respond to a humanistic act integrating the reality of each student with the purpose of education, which leads to the free and independent formation of each being.
\end{abstract}

Keywords: educational reality; rural schools; curricular decontextualization; rural teaching performance.

How to cite this essay:

Hernández, A. (2021). Approach to the educational reality in schools in the Venezuelan rural sector. Revista Scientific, 6(19), 264-278, e-ISSN: 2542-2987. Recovered from: https://doi.org/10.29394/Scientific.issn.2542-2987.2021.6.19.13.264-278

Date Received: 11-09-2020
Date Acceptance:

04-12-2020
Date Publication:

05-02-2021 


\section{Ensayo Original / Original Essay}

\section{Introducción}

La realidad educativa del sector rural venezolano muestra que el proceso de enseñanza y aprendizaje se realiza con prácticas pedagógicas no acordes, al aplicar programas educativos escasamente cónsonos con el contexto rural; el trabajo docente en condiciones rurales, implica atender simultáneamente niños y niñas de diversos grados, edades, con diferentes intereses y necesidades, lo cual significa que la organización escolar debe responder a una forma particular.

Por consiguiente, la constitución heterogénea de los grupos en el aula de clase permite al docente favorecer la colaboración, ayuda mutua y trabajo en equipó entre sus estudiantes, pero al mismo tiempo le demanda organizar el trabajo, articulando los contenidos de los diferentes grados, evitando la fragmentación del proceso de aprendizaje, atendiendo a las particularidades y realidades del contexto rural.

De acuerdo con lo anterior, el abordaje del proceso educativo en las escuelas del sector rural, debe ser visto de una manera especial, el interés tiene que estar centrado en el ámbito socio cultural del contexto, con un estilo de enseñanza orientado a la diferenciación de los niveles de aprendizaje de cada estudiante, que conduzca la formación de un ser crítico, capaz de transformar su realidad; en consecuencia la orientación de las políticas educativas para este sector deberían adecuarse a las condiciones económicas, políticas y sociales del contexto.

Desde esta perspectiva es necesario, la revisión y adecuación de las políticas educativas para el sector rural, en el cual se considere la cultura en el entorno como parte fundamental del ambiente en donde se desarrolla la actividad humana de la educación; además de ello, el docente tiene que ser un ente activo en la discusión del impacto que debe generar la educación en la sociedad.

En este orden de ideas, el papel del docente debe estar enmarcado en 
desarrollar un trabajo en conjunto con las personas que forman la comunidad educativa para actuar de manera positiva y colaborativa. Bajo estas consideraciones, la escuela en el sector rural tiene la necesidad de abrirse al contexto, a las familias, al estudiantado, por ello, el proceso de enseñanza y aprendizaje debe partir desde el entorno, las tradiciones, costumbres y la comunidad en general, todo integrado dentro del currículo.

En razón de lo anterior, la educación que se necesita hoy en el medio rural para enfrentar los retos que se imponen constantemente por los cambios dentro de la sociedad, debe ser una educación que se desprenda de todo tipo de formalismo y dogmatismo, que no exista ruptura entre el pensamiento y el lenguaje, en el cual los estudiantes tengan libertad para que expresen lo que piensan y sienten.

Por consiguiente, existe la necesidad de convertir el proceso de enseñanza y aprendizaje del sector rural en el medio para orientar al estudiante a aprovechar su capacidad física, asumiendo valores, normas, realizando uso racional de los recursos naturales que le rodean, desarrollando arraigo y sentido de pertinencia por su comunidad. Por tanto, se debe evitar crearles espejismos que lo obliguen sutilmente dejar su contexto, para introducirse en ese mundo imaginario que le han venido creando los programas de estudio, en relación a la vida en la ciudad que allí se les presenta de forma maravillosa.

\section{Desarrollo}

La educación en las escuelas del sector rural venezolano ha existido con una práctica formal desde épocas muy remotas, sin embargo, a lo largo de la historia de las políticas educativas, se puede ver hoy, en confrontación con la realidad del campesino venezolano como estuvo y continúa desvinculada del contexto, impidiendo la pertinencia socio-cultural de la educación con el sector rural. 
En este sentido, la función de la educación es lograr el desarrollo integral del individuo, esté ubicado en medio urbano o rural, para alcanzar este fin se tiene que establecer una relación de ayuda y colaboración entre padres, madres, docentes, estudiantes y comunidad en general, mediante un trabajo en equipo que permita el impulso de las potencialidades, habilidades, destrezas y conocimientos en los estudiantes.

En atención a lo antes expuesto, se hace necesario cada vez analizar con mayor énfasis, cómo transformar el paradigma de enseñanza tradicional para hacerlo más eficiente sobre todo en lo que respecta a la educación rural. De igual manera, se hace imprescindible lograr una verdadera integración entre la escuela, la familia y la comunidad, como elemento fundamental para el desarrollo de un modelo pedagógico de calidad centrado en el estudiante.

En correspondencia, López (2006): refiere a "los habitantes del mundo rural, por sus condiciones de aislamiento, dispersión, su relación con el medio natural, las ocupaciones que desarrollan, sus formas particulares de vivienda y organización social, requieren una educación que dé respuesta a sus particularidades poblacionales" (pág. 154).

Infiriendo sobre lo expresado por el autor, la preocupación por mejorar la calidad con relación a la educación del sector rural, conduce a pensar en las formas que faciliten la organización del proceso de enseñanza y aprendizaje, pues requiere de un tratamiento especial; que reivindique saberes y necesidades locales, que nazca desde los intereses del mundo rural.

Consecuentemente, la distribución del estudiantado de diversos grados en una misma aula de clase, es una característica que hay que tener presente; esta organización se ha denominado aulas multigrados o escuelas unitarias, en el cual estudiantes de distintos grados comparten la clase. Esta peculiaridad dificulta la tarea de muchos docentes que no consiguen la manera de enseñar a la vez niños de distintas edades, grados, intereses y necesidades, pues es algo que en la carrera profesional no les han enseñado. 
Por otra parte, este tipo de organización escolar en la cual estudiantes de distintos grados, comparten una misma aula con un solo docente genera una capacidad de ayuda y colaboración. Este aspecto, debe ser aprovechado por el docente en el proceso de aprendizaje para fortalecer valores, la cooperación y el compañerismo, provocando en los estudiantes el conocimiento, la relación entre ellos, la motivación para trabajar en equipo y ayuda a la hora de aprender, promover la participación en la toma de decisiones consensuadas, revisar el funcionamiento del grupo y la autoevaluación. Al respecto, García (2015): manifiesta que en este tipo de aula se genera "una convivencia participativa, en una gestión compartida del aula. De este modo, la convivencia no solo es una cuestión del docente, también están implicados los escolares [...]" (pág. 100).

Asimismo, las aulas multigrado, requieren de mayor atención individualizada y la personalización de la enseñanza para un mejor desarrollo de las capacidades, he aquí la importancia de preguntarse ¿cómo realizar ese tipo de enseñanza personalizada con la diversidad presente en el aula, que no conlleve a la desatención de un estudiante u otro?, teniendo presente ¿cómo aprende?; ¿cómo es consciente de su aprendizaje?; ¿y qué debe hacer para mejorarlo?, es decir, hay que fijarse en las capacidades individuales para desarrollarlas al máximo.

Lo anteriormente expresado, conduce a reflexionar acerca de que la enseñanza en las escuelas del sector rural debe responder a un acto humanista que integre la realidad de cada estudiante con el propósito de la educación, que conduzca a la formación libre e independiente de cada ser, en el cual la solidaridad, la colaboración, cooperación y trabajo en equipo entre docentes, familias y comunidad sea esa fuerza del despertar de la conciencia que conlleve a un mundo más humano y digno.

En este orden de ideas, la descontextualización curricular es otra de las realidades a las que se enfrenta la educación en los entornos rurales, 
generando la desintegración y desvinculación de los contenidos enseñados. Señalando a Núñez (2010): "los conocimientos enseñados en la escuela rural, en su mayoría, son abstractos, fragmentados y desvinculados de la vida y del trabajo" (pág. 3); ocasionando el bajo rendimiento escolar, la repitencia y deserción. De allí la importancia de integrar las prácticas educativas con el contexto en el cual se desenvuelven los estudiantes para generar aprendizajes útiles y aplicables en el contexto rural.

Es por ello, que se deben aprovechan los recursos del entorno para alcanzar los propósitos de la tarea educativa en el sector rural, asumiendo las practicas escolares tanto individualizada como colectiva, de manera flexible y adaptadas a las necesidades, culturas y contextos de los estudiantes, generando conocimientos teóricos y prácticos, valores y actitudes necesarias para convivir en sociedad, desarrollando capacidades para vivir y trabajar con dignidad, participando plenamente en el desarrollo de las comunidades y mejorando la calidad de vida.

En correspondencia con las convicciones antes señaladas, para evidenciar el logro de las metas educativas, fortalecer los vínculos institucionales y comunitarios, es decir, para hablar de calidad educativa en entornos rurales es necesario considerar diversos elementos en la organización del proceso escolar. En este sentido, Ávila (2017), plantea que:

Existen elementos, cuyos valores de efectividad están intrínsecos como la pertinencia del currículo, la participación de la comunidad en la construcción y desarrollo del mismo, la visión comunitaria, la inclusión de los menos favorecidos en programas de desarrollo local, la metodología en el proceso de enseñanza (pág. 94).

En este sentido, es necesario la construcción de un currículo flexible propio para el sector rural, mediante la participación y cooperación de las comunidades, en el cual se plantee la enseñanza como un proceso holístico en el que se relacionen los contenidos curriculares con las experiencias de 


\section{Ensayo Original / Original Essay}

acuerdo al contexto, pues al estudiante construir su aprendizaje partiendo de los saberes y la realidad que lo envuelve comprenderá la utilidad y aplicabilidad en la vida diaria y por ende en el contexto rural.

En efecto, para que un conocimiento sea relevante la práctica docente y el desarrollo del currículo general deben unificarse, junto a materiales curriculares contextualizados que medien ese proceso y favorezcan la reflexión, al igual que la toma de decisiones respecto a las estrategias y actividades de enseñanza que utilizará el docente en función de las características de su grupo de estudiantes.

Es importante acotar, que la escuela en el sector rural representa un espacio para la innovación pedagógica en función de los requerimientos de las comunidades, para ello, se requieren políticas educativas enmarcadas en la contextualización curricular, que coadyuven en la organización del proceso escolar para la ruralidad, incorporando las experiencias culturales, sociales, económicas, domésticas y productivas en el desarrollo de los programas y contenidos, lo cual permitirá contar con un currículo, tal como lo plantean Contreras y Mujica (2014): es "[...] permeable, flexible, constructivo y con pertinencia social" (pág. 44).

Cabe agregar que la realidad de la educación rural refleja como el proceso de enseñanza se realiza con estrategias no acordes a las necesidades e intereses de los estudiantes lo que genera desmotivación y falta de interés. Por ello, es necesario seleccionar los contenidos apropiados utilizando materiales curriculares que medien el proceso de enseñanza y aprendizaje; de esta manera, se estaría proporcionando a los estudiantes del sector rural igualdad de oportunidades educativas, previendo atención diferenciada a las del contexto urbano.

Acorde con esto, Arrieche (2018), manifiesta que: "es necesario una estrecha relación entre las instituciones educativas y su entorno político, social y económico, en donde debe actualizarse diseños curriculares adecuados a 


\section{Ensayo Original / Original Essay}

las necesidades del individuo y la sociedad [...]" (pág. 356). De esta manera se forma un ciudadano integro, capaz de enfrentar los cambios que surgen en la sociedad y resolver los problemas que se le presenten en el medio rural.

Otro de los aspectos que caracterizan la realidad de la educación rural, es la ausencia de formación docente para su desempeño en condiciones rurales; aunado a ello, la universidad tampoco forma ni prepara, pues, se ajusta a los parámetros de una educación globalizada, donde el conocimiento se imparte de manera general, con los mismos contenidos, dinámicas y metodologías de enseñanza para todos por igual, olvidando que en la praxis las realidades contextuales son completamente diferentes, tal como ocurre con la educación rural donde el ejercicio profesional e caracteriza por encontrar grupos de estudiantes en una misma aula con edades diferentes, cursando grados diferentes, pero formando parte de una dinámica sistematizada. Al respecto, Peirano, Estévez y Astorga (2015), describen:

La presencia de escuelas multigrado y el aislamiento geográfico en la mayoría de las instituciones educativas rurales trae como consecuencia que muy pocos docentes o incluso uno solo deban atender las características y necesidades individuales de alumnos con distintas edades y niveles de conocimiento. Esta realidad los obliga a conocer y a utilizar estrategias pedagógicas que les permitan guiar a un grupo diverso de estudiantes en sus trayectorias de aprendizaje (pág. $67)$.

Es decir, el docente que labora en el sector rural debe estar bien preparado, ya que corresponde a una realidad educativa donde la heterogeneidad se hace presente en la vida cotidiana y requiere organizar los procesos pedagógicos de manera que cubra las necesidades de enseñanza de manera particular.

A su vez, Ramírez-González (2015a): sostiene que "la labor pedagógica debe adaptarse a los cambios que las zonas rurales enfrentan, por lo que la planificación de sus actividades requiere de docentes rurales con una posición 


\section{Ensayo Original / Original Essay}

flexible, proactiva, real y útil para el estudiantado [...]” (pág. 7). Además de ello, el docente de estas escuelas cumple funciones pedagógicas y administrativas, debido a que en ocasiones es el único en la escuela y le corresponde organizar su tiempo para cumplir con ambas tareas.

Por otro lado, Ramírez-González (2015b), en miras de minimizar los conflictos que a menudo enfrenta el docente estable un perfil que apuntala hacia la realidad de las zonas rurales:

Un perfil docente rural retoma aspectos de la ocupación, del desempeño, sus cualidades y las características de su personalidad en relación con miembros de la comunidad, así como de la formación profesional para la docencia en la ruralidad, por lo que su práctica educativa se configura a partir de características profesionales de las dimensiones académica, personal, ocupacional, fundamentadas en la interacción con la comunidad, los padres y madres de familia y otras instancias gubernamentales o no, influyentes en los procesos educativos (pág. 8).

Por tanto, el perfil del docente de las escuelas del sector rural requiere de una formación pedagógica y gerencialmente, pues son muchas las competencias que debe abarcar, siendo necesario conocer al estudiante para determinar intereses y necesidades en función de las cuales se organiza la planificación del quehacer educativo.

De esta manera, se podría afirmar entonces que las competencias pedagógicas que debe desarrollar un docente están relacionadas con su capacidad para poder hacer del aula de clase un lugar de innovación y emprendimiento donde los estudiantes logren desarrollar sus capacidades de lectura, análisis, interpretación, reflexión, discusión, en otras palabras, que desarrollen el pensamiento crítico, junto a ello, es importante que actúen con responsabilidad, motivación e interés en su proceso de aprendizaje, promoviendo de esta manera un aprendizaje significativo.

Al respecto, Molina (2019): plantea que "se requiere que las 
instituciones formadoras brinden herramientas pedagógicas que fortalezcan las competencias creativas y recursivas de los futuros educadores, siempre y cuando éstas sean exigidas como respuesta a las condiciones laborales que deben enfrentar" (pág. 99). A su vez, el docente, debe desarrollar competencias investigativas, que fortalezcan su profesión para conducir con propiedad el desarrollo integral de los estudiantes, logrando solventar los problemas que se van suscitando en su quehacer diario, cooperado con la transformación y modificación de situaciones para propiciar el buen funcionamiento de las instituciones educativas.

\section{Conclusiones}

La praxis pedagógica en entornos rurales requiere de un docente que se mantenga en un constante proceso de reflexión en cuanto a la forma de estructurar el proceso de enseñanza y aprendizaje; desde donde partir, como organizar los programas, libros de texto, material de apoyo y currículo en general que le permita atender los intereses y necesidades de los estudiantes, distribuyendo el tiempo equitativamente para cada grado, como encontrar ese punto de convergencia entre los distintos contenidos para alcanzar una enseñanza contextualizada.

Bajo este contexto, es necesario hablar de renovación pedagógica, en las escuelas del sector rural, se debe constituir en un componente fundamental de una educación contextualizada, centrada en el estudiante y preocupada por ampliar las posibilidades de interacción entre niños de diversas edades, de allí la necesidad de repensar la enseñanza para utilizar una metodología apropiada. Esto indica que aún hay mucho por hacer en términos de una pedagogía propia para el entorno rural: los estímulos para el auto aprendizaje, la atención individualizada, el trabajo en grupo, el interaprendizaje y la organización de contenidos.

De igual manera, la integración de todos los actores de la comunidad 


\section{Ensayo Original / Original Essay}

educativa es fundamental, pues favorece la participación activa de las familias y comunidades en el proceso de enseñanza y aprendizaje, permitiendo mejorar la calidad educativa a través del logro de los objetivos institucionales. Por ello, el docente del sector rural debe convertirse en líder, promoviendo el bienestar social y la solución de los problemas que subyacen en el entorno escolar.

Efectivamente, las prácticas pedagógicas en entornos rurales haciendo uso de un currículo flexible, facilitan el trabajo sistemático, colaborativo y cooperativo entre los diferentes actores educativos (docentes, estudiantes, comunidad educativa y comunidad en general), sin embargo, el mayor beneficio está orientado hacia los estudiantes quienes adquieren los conocimientos teóricos que irán de la mano con la demostración práctica, cambiando paradigmas y fortaleciendo competencias que los libros no contienen. Además, promueve la integración de saberes en cualquier contexto.

Efectivamente, las políticas educativas para el sector rural se deben enfocar en la construcción de un currículo adaptado a las características y realidades propias del campo, que promueva una enseñanza contextualizada, con utilidad y aplicabilidad en la vida cotidiana para contribuir en el mejoramiento de las condiciones de vida de los habitantes, dando solución a las diferentes problemáticas que afronta este sector.

En atención a ello, los docentes necesitan buscar un conocimiento profundo de los materiales y desarrollo curricular para incorporar metodologías propias y adecuadas al contexto rural, en la cual se reconozca, valore y revalorice las culturas rurales equilibrando los saberes locales con el saber universal de cada disciplina o área del conocimiento, mediante un acomodamiento contextual e integración curricular en la escuela rural.

En correspondencia con lo anterior, el estado venezolano debe crear programas de formación para docentes rurales, permitiendo el crecimiento de un profesional con sólidas competencias personales y pedagógicas, para 
impulsar la educación en los sectores rurales, con capacidad para contextualizar la práctica pedagógica, promoviendo un currículo que responda a las necesidades en la cual se encuentra inserta la escuela, favoreciendo el sentido de identidad personal y cultural de los estudiantes, propiciando un sentimiento de arraigo de los habitantes con respecto al contexto rural en el que vive.

\section{Referencias}

Arrieche, M. (2018). Gestión Docente en el Contexto de la Educación Primaria Venezolana. Revista Scientific, 3(7), 354-373, e-ISSN: 25422987. Recuperado de: https://doi.org/10.29394/Scientific.issn.2542$\underline{2987.2018 .3 .7 .18 .354-373}$

Ávila, B. (2017). Aportes a la calidad de la educación rural en Colombia, Brasil y México: experiencias pedagógicas significativas. Tesis doctoral. Bogotá, Colombia: Universidad de la Salle. Recuperado de: https://ciencia.lasalle.edu.co/doct educacion sociedad/12

Contreras, W., \& Mojica, G. (2014). El docente del sector rural y sus competencias: Aproximación a la construcción de un perfil de competencias para el docente que labora en el sector rural. 1ra. edición. Caracas, Venezuela: Publicaciones de la Universidad Pedagógica Experimental Libertador.

García, F. (2015). Escuela, medio rural y diversidad cultural en un contexto global: currículum, materiales didácticos y práctica docente de Conocimiento del Medio: situación, límites y posibilidades en centros onubenses. Tesis doctoral. Huelva, España: Universidad de Huelva. Recuperado de:

http://hdl.handle.net/10272/11440

López, L. (2006). Ruralidad y educación rural. Referentes para un Programa de Educación Rural en la Universidad Pedagógica 
Nacional. Revista Colombiana de Educación, (51), 138-159, e-ISSN:

0120-3916. Recuperado de:

https://www.redalyc.org/articulo.oa?id=413635245006

Molina, B. (2019). El quehacer del maestro y la formación docente en la escuela rural multigrado. Trabajo de grado. Tunja, Colombia: Universidad Pedagógica y Tecnológica de Colombia. Recuperado de: https://repositorio.uptc.edu.co/handle/001/2728

Núñez, J. (2010). Pertinencia de la educación rural venezolana y latinoamericana. Revista Iberoamericana de Educación, 52(7), 1-14, e-ISSN: 1681-5653. Recuperado de:

https://doi.org/10.35362/rie5271762

Peirano, C., Estévez, S., \& Astorga, M. (2015). Educación rural: oportunidades para la innovación. Cuadernos de Investigación Educativa, 6(1), 53-70, e-ISSN: 1688-9304. Recuperado de: https://dialnet.unirioja.es/servlet/articulo?codigo $=5367416$

Ramírez-González, A. (2015a,b). Valoración del perfil docente rural desde el proceso formativo y la práctica educativa. Revista Electrónica Educare, 19(3), 1-26, e-ISSN: 1409-4258. Recuperado de:

http://dx.doi.org/1015359/ree.19-39 


\section{Adriana Hernández Lobo}

e-mail: fatimavalentina 22@hotmail.com

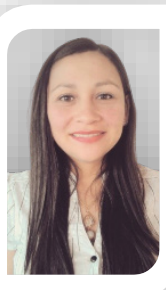

Nacida en Mérida estado Mérida, Venezuela el 22 de junio del año 1988. Licenciada en Educación egresada de la Universidad Bolivariana de Venezuela (UBV); Especialista en Gerencia Educacional en la Universidad Pedagógica Experimental Libertador (UPEL); investigadora en Constructos Teóricos emergentes sobre la vinculación de la Pedagogía Integradora con el desempeño docente en el contexto de la Educación Rural Venezolana; Docente de aula multigrado en el sector rural municipio Aricagua del estado Mérida; facilitadora de seminario I en la maestría de Gerencia Educativa de la Universidad Pedagógica Experimental Libertador. 